\title{
Increase in NF-кB-sensitive miRNA-146a and miRNA-155 in multiple sclerosis (MS) and pro-inflammatory neurodegeneration
}

\author{
Deidre J. Devier ${ }^{1,2}$, Jesus F. Lovera² and Walter J. Lukiw ${ }^{2,3,4 *}$ \\ ${ }^{1}$ Department of Cell Biology and Anatomy, Louisiana State University Health Sciences Center, New Orleans, LA, USA \\ 2 Department of Neurology, Louisiana State University Health Sciences Center, New Orleans, LA, USA \\ ${ }^{3}$ Neuroscience Center of Excellence, Louisiana State University Health Sciences Center, New Orleans, LA, USA \\ ${ }^{4}$ Department of Ophthalmology, Louisiana State University Health Sciences Center, New Orleans, LA, USA \\ *Correspondence: wlukiw@lsuhsc.edu
}

Edited by:

Jun Yan, The University of Queensland, Australia

Reviewed by:

Daniele Bottai, University of Milan, Italy

Chiara Fenoglio, University of Milan, Italy

Keywords: autoimmunity, degeneration, multiple sclerosis, microRNA (miRNA), miRNA-146a, miRNA-155, inflammation, NF-kB (p50/p65)

\section{OVERVIEW}

Multiple sclerosis (MS) is a complex, debilitating, immunopathologic disease of the human central nervous system (CNS) characterized by chronic systemic inflammation, alterations in innate-immune signaling, progressive demyelination and axonal loss. Currently there is no effective treatment or cure for MS. Recent data indicate that common molecular-genetic mechanisms involving a select group of NF-kB-sensitive microRNAs are shared by most MS patients, and their mechanism of pathogenic action is becoming increasingly understood. This brief "Opinion" paper will highlight some recently clarified roles for two NF-kBregulated, pro-inflammatory microRNAs, miRNA-146a and miRNA-155, in the MS disease process. We will also advance an opinion on how anti-NF-kB, antimiRNA and/or related therapeutic strategies may be beneficial in the clinical management of MS and other progressive CNS diseases exhibiting inflammatory neurodegeneration.

\section{MULTIPLE SCLEROSIS—INCIDENCE, SYMPTOMS, AND AUTOIMMUNITY}

To understand the contribution of miRNA-146a and miRNA-155 to the etiopathogenesis of MS we will briefly highlight some salient features of this autoimmune disease. MS is globally the most common immunopathologic disorder affecting the human CNS: (i) with about 2.5 million affected worldwide (approximately 30 cases $/ 100,000$ globally; http://www.nationalmssociety.org/); (ii) with a variable incidence of frequency in different regions of the world (Melcon et al., 2014); (iii) with a variable incidence of occurrence amongst different human populations (Høglund and Maghazachi, 2014); (iv) with significant heterogeneity in the clinical phenotype (Sturm et al., 2014; http://www. nationalmssociety.org/); and (v) with low concordance rates in monozygotic twins, suggesting the involvement of complex heritable, epigenetic, microbial and/or environmental factors (Küçükali et al., 2014; Ma et al., 2014; Melcon et al., 2014). MS is generally characterized by abnormal responses of the immune system directed against glial-derived CNS myelin which normally sheaths, insulates and protects axons and nerve bundles (Küçükali et al., 2014; Melcon et al., 2014). MS exhibits (i) "sclerotic" or lipoprotein-enriched scar tissue nodules associated with demyelinated axons where normal electrical activities become progressively disorganized (Baranzini, 2014; Küçükali et al., 2014); and (ii) a wide variety of clinical symptoms that include muscle weakness, spasticity, loss of balance, sensory deficit and fatigue, dizziness, and vertigo, bowel, bladder and visual problems, depression and cognitive and psychiatric changes; approximately $90 \%$ of MS individuals become ultimately disabled (Baranzini, 2014; Guo et al., 2014; Harris and Sadiq, 2014). Consistent observations at the pathogenic and molecular-genetic level indicate five main highly interactive characteristics of MS: (i) a progressive demyelination whose extent correlates to MS severity; (ii) axonal swelling and macrophage activation; (iii) a T-cell mediated inflammatory response that subsequently triggers immune cells to release pro-inflammatory cytokines such as IL-1 $\beta$; (iv) permeability changes in the blood-brain barrier (Kamphuis et al., 2015); and (v) increases in proinflammatory microRNA and related pathogenic biomarkers (Haghikia et al., 2012; Meinl and Meister, 2012; Danborg et al., 2014; Harris and Sadiq, 2014; Küçükali et al., 2014; Ma et al., 2014; Sturm et al., 2014; Kamphuis et al., 2015; see below). Specific gene mutations linked to MS include a cluster at human chromosome 6, part of the so-called "autoimmunome" network, which serves as the major histocompatibility complex (MHC) locus; interestingly this genetic locus is also implicated in the autoimmune disease type 1 diabetes and systemic lupus erythematosis (Baranzini, 2014; Gourraud et al., 2014; Sturm et al., 2014). Epidemiological evidence collectively indicates that MS is an immunopathologic disorder initiated by exogenous factors including microbes, possibly of viral origin, vaccines or unknown environmental factors in susceptible individuals genetically predisposed for MS (Gilden, 2005; 
Küçükali et al., 2014; Ma et al., 2014; Sturm et al., 2014). Indeed, heterogeneity in the MS clinical course and low twin concordance rates implicate multiple, complex, environmental and epigenetic factors that contribute to MS pathogenesis and most recently, a potential contribution by inducible species of CNS microRNAs (Haghikia et al., 2012; Lopez-Ramirez et al., 2014; Ma et al., 2014; Zhang et al., 2014; Kroesen et al., 2015).

\section{microRNA (miRNA) UP-REGULATION AND TARGET MRNA DOWN-REGULATION}

Human CNS microRNAs (miRNAs) constitute a family of about 2050, 20-23 nucleotide non-coding single stranded RNAs (ssRNAs) that regulate the expression of their target mRNAs post-transcriptionally, and have important roles in development, differentiation, aging, autoimmunity and neurodegeneration (Lukiw et al., 2008; Cui et al., 2010; Guo et al., 2010; O'Connell et al., 2010; Li et al., 2011; Zhang et al., 2014; Zhao et al., 2014).

One major mode of action in the CNS is for inducible, up-regulated miRNAs to decrease their target mRNA levels and hence decrease gene expression (Cui et al., 2010; Guo et al., 2010). Concurrent induction of both NF-kB (p50/p65) and pro-inflammatory miRNAs in stressed human primary brain cells has identified a group of pathogenic miRNAs under NF$\mathrm{kB}$ (p50/p65) transcriptional control and these include miRNA-146a and miRNA155 (Lukiw, 2012a; Zhang et al., 2014; Zhao et al., 2014). Interestingly, these same miRNAs have been found to be increased in sporadic Alzheimer's disease (AD) tissues which exhibit (i) significant global up-regulation of NF-kB in $\mathrm{AD}$-affected anatomical regions (Lukiw and Bazan, 1998; Lukiw, 2012b); (ii) down-regulation in the expression of innate-immune markers such as the IL- $1 \beta$ receptor-associated kinase 1 (IRAK-1; with a concurrent surge in IRAK-2; Cui et al., 2010); and (iii) a progressive inflammatory degeneration (Latta et al., 2014). Different species of pro-inflammatory miRNAs in different CNS compartments may contribute to similar degenerative pathologies - for example miRNA-146a and miRNA155 have slightly different effects on inflammatory gene expression in human brain and retina (see below; Lukiw et al., 2012; Ma et al., 2014). It is also important to point out that there appears to be some heterogeneity in miRNA abundance, complexity and related biomarkers amongst different human populations with the same neurological disorder, however recent evidence suggests important common, underlying pathogenic roles for miRNA-146a and miRNA-155 throughout the MS disease process (Meinl and Meister, 2012; Kutty et al., 2013; Harris and Sadiq, 2014; Ma et al., 2014).

\section{miRNA-146A AND INFLAMMATORY DEGENERATION}

Few CNS-resident miRNAs have gained so much interest as an inducible "potentially pathogenic miRNA" implicated in NF$\mathrm{kB}$-mediated pro-inflammatory signaling and aberrant activation of the innateimmune response as has miRNA-146a, in a surprisingly wide variety of progressive, neurodegenerative CNS disorders. These include AD, MS, and prion-, neurotropic virus- and metal-sulfate-induced neurological dysfunctions (Taganov et al., 2006; Lukiw et al., 2008; Hill et al., 2009; Pogue et al., 2009; Cui et al., 2010; Saba et al., 2012; Kutty et al., 2013; Ma et al., 2014). The 22 nucleotide miRNA-146a (5'-UGAGAACUGAAUUCCAUGGGUU3'; 59\% A + U; NCBI Gene ID: 406938), encoded at human chromosome $5 \mathrm{q} 33.3$ is a rapidly induced, pro-inflammatory miRNA with a relatively short half-life of about $1.5-2 \mathrm{~h}$ in human CNS cells and tissues (Sethi and Lukiw, 2009). miRNA146a was originally described as being significantly up-regulated after microbial endotoxin, lipopolysaccharide or cytokine stimulation of myeloid cells and under transcriptional control by NF- $\mathrm{B}$; shortly thereafter this inducible miRNA146 a was found to be up-regulated by metal sulfate-generated reactive oxygen species, by pro-inflammatory cytokines (such as IL-1 $\beta$ and $\mathrm{TNF} \alpha$ ) and by $\mathrm{A} \beta 42$ peptides in human primary brain cells (Taganov et al., 2006; Lukiw et al., 2008; Hill et al., 2009; Pogue et al., 2009; Cui et al., 2010). Further, miRNA-146a targets the $3^{\prime}$-UTR of mRNAs encoding signaling proteins involved in the innate immune and inflammatory response, including complement factor $\mathrm{H}(\mathrm{CFH})$ and IRAK-1, and both compartmentalized CFH and IRAK-1 deficiencies are observed in MS (Taganov et al., 2006; Cui et al., 2010; Ingram et al., 2014; Stürner et al., 2014). Interestingly, significant amounts of miRNA-146a have been found in glial cells responsible for axonal myelination (Li et al., 2011; Alexandrov et al., 2014; Kroesen et al., 2015). Of further interest is that viral infections of brain cells by herpes simplex virus 1 (HSV-1; Herpesviridae; Group 1 dsDNA) and other neurotrophic viruses both up-regulate NF-kB and miRNA-146a, and induce pathogenic events that can be quenched using antiviral drugs such as acyclovir (Hill et al., 2009; Lukiw et al., 2010).

\section{miRNA-155 AND NEURODEGENERATIVE DISEASE}

The 23 nucleotide miRNA-155 (5'UUAAUGCUAAUCGUGAUAGGGGU-3'; 61\% A + U; NCBI: AF402776) encoded in humans at chr $21 \mathrm{q} 21.3$ is an inducible miRNA under transcriptional control by NF-kB (p50/p65; Lukiw et al., 2012; Lu et al., 2013). miRNA-155, strongly and rapidly up-regulated by inflammatory cytokines, is highly expressed within lymphocytes (both $\mathrm{B}$ and $\mathrm{T}$ cells) and its role in adaptive and innate immunity has also been strengthened by recent evidence (Lukiw, 2012a,b; Lopez-Ramirez et al., 2014; Seddiki et al., 2014). Specific up-regulation of miRNA-155 is observed in related immunopathologic conditions including MS, Down's syndrome (trisomy 21), rheumatoid arthritis and systemic lupus erythematosus where it affects both $\mathrm{T}$ lymphocyte and blood-brain barrier functions (Junker, 2011; Leng et al., 2011; Li et al., 2012; Lopez-Ramirez et al., 2014; Kamphuis et al., 2015). Compared to controls miRNA-155 is the only miRNA commonly increased in MS brain, spinal cord lesions and in peripheral blood mononuclear cells (PBMC; $\mathrm{Ma}$ et al., 2014). Interestingly, in experimental autoimmune encephalomyelitis (EAE), a mouse model for MS, miRNA155 expression has been shown to increase significantly in the spleen, lymph node and brain as EAE progresses, and miRNA155(-/-) mice exhibit resistance to EAE and significantly less CNS inflammation (Murugaiyan et al., 2011; Thamilarasan et al., 2012). Both miRNA-146a (see 
above) and/or miRNA-155: (i) target the CD47 3'-UTR, promoting downregulation of $\mathrm{CD} 47$ on brain resident cells, triggering the macrophage-mediated phagocytosis of myelin (Junker et al., 2009) and/or (ii) induce the development of IFN- $\gamma$-producing T helper type 1 (Th1) cell subsets and CD4(+) T cells that secrete interleukin (IL)-17 (Th17 cells); these cells are known to induce inflammatory degeneration in MS, psoriasis, autoimmune uveitis and rheumatoid arthritis (Ma et al., 2014; Seddiki et al., 2014; Stürner et al., 2014). Very recently it has been shown that (i) suppressing miRNA-155 expression inhibits the development of Th1 and Th17 cells, resulting in the decrease of disease severity in EAE (Zhang et al., 2014); and (ii) anti-miRNA-155 treatment in EAE significantly inhibits EAE development (Murugaiyan et al., 2011; Ma et al., 2014; Seddiki et al., 2014). As miRNA-155 is encoded on chr21q21.3, the extra gene dosage of chromosome 21 in trisomy 21 patients is associated with (i) increased miRNA-155 levels; (ii) proinflammatory signaling and deficits in the innate immune response; and (iii) an increased incidence of autoimmune disease with cognitive disabilities ( $\mathrm{Lu}$ et al., 2008; Li et al., 2012). The up-regulation of CNS miRNA-155 has also been associated with infection by Bornavirus (Bornaviridae; Group V, negativesense ssRNA) that causes an enzootic encephalomyelitis, related to MS with symptoms that include ataxia, ocular disorders, abnormal posture and movement impairment, neurologic disturbances and cognitive/psychiatric disturbances (http://www.malacards.org/card/borna_dise ase?search $=$ borna + disease $)$.

\section{ANTI-miRNA vs. anti-NF-kB STRATEGIES}

In recent efforts to neutralize NF-kBtriggered, miRNA-mediated pathologies, the use of anti-miRNA and anti-NF-kB strategies have worked surprisingly well both in vitro and in vivo in animal experimentation. For example anti-miRNA-146a and/or anti-miRNA-155 LNA-protected oligonucleotides administered individually or as combinatorial cocktails exhibited significant efficacy in cytokine-stressed human primary neuronal-glial cell cocultures and in EAE in reducing aberrant
AD- and MS-related pro-inflammatory signaling (Cui et al., 2010; Murugaiyan et al., 2011; Lukiw et al., 2012; LopezRamirez et al., 2014). Very recently miRNA-155 up-regulation that altered junctional organization and permeability of the blood-brain barrier in MS murine models was prevented using inhibition of endogenous miRNA-155 (Lopez-Ramirez et al., 2014; Kamphuis et al., 2015). Equally efficacious appear to be the use of anti-NF$\mathrm{kB}$ remedial strategies; the current number of NF-kB inhibitors now exceeds 900 and the use of combined anti-miRNA and NF$\mathrm{kB}$ inhibitors, and how and when to use them therapeutically, has been recently addressed (Gilmore and Herscovitch, 2006; Lukiw, 2012a,b, 2013; Gibson, 2014). In our view pathogenic miRNA146a and miRNA-155 up-regulation in several progressive immunodeficiency and/or pro-inflammatory disorders of the CNS indicates that (i) knowledge of the disease mechanism in one neurological disorder may shed some light on a similar disease mechanism in a related CNS disease; (ii) differential anti-miRNA and/or anti-NF-kB therapeutic strategies, perhaps using combinatorial cocktails, should be useful in the clinical management of neurological disorders such as MS; and (iii) multiple NF-kB inhibitors, perhaps combined with multiple antimiRNA oligonucleotides and current MS pharmacological drugs including dimethyl fumarate and steroids may be tailored to suit each individual MS case in the expanding arena of personalized medicine (Lukiw, 2012a,b; Gotovac et al., 2014; Harris and Sadiq, 2014; Latta et al., 2014).

\section{CONCLUDING REMARKS}

Our understanding of NF-kB-regulated miRNAs and their abundance and complexity are revolutionizing our perceptions and ideas on gene expression in CNS aging and disease. It is our opinion that (i) progressive inflammatory neurodegeneration in MS involves NF- $k B$-regulated miRNA$146 a$ and miRNA-155 and perhaps other pathogenic miRNAs which are inducible; (ii) targeting of inflammation-relevant gene expression by miRNA-146a and/or miRNA155 suggest pathogenic pathways of MS may be in common with other kinds of human CNS degenerations; (iii) altered miRNA expression patterns in MS may be useful both diagnostically and in the design of novel therapeutic approaches; and (iv) anti-miRNA-146a, anti-miRNA-155, anti$N F-k B$ and perhaps anti-viral drugs, either alone or in combination with currently prescribed MS anti-inflammatory drugs, should open new avenues for future MS research and therapeutic strategies useful in the clinical management of MS and other CNS disorders with a progressive inflammatory and degenerative component.

\section{ACKNOWLEDGMENTS}

This research was presented in part at the Society for Neuroscience (SFN) Annual Meeting, San Diego CA, USA, 9-13 November 2013. Research in the Lukiw laboratory on microRNA, non-coding single stranded RNA (ssRNA), the innateimmune response, amyloidogenesis, and neuroinflammation in $\mathrm{AD}$, retinal and prion disease using post-mortem brain tissues, was supported through a COBRE III Pilot Award, an unrestricted grant from Research to Prevent Blindness (RPB), the Louisiana Biotechnology Research Network (LBRN), and NIH grants NEI EY006311 and NIA AG038834.

\section{REFERENCES}

Alexandrov, P. N., Dua, P., and Lukiw, W. J. (2014). Up-Regulation of miRNA-146a in progressive, age-related inflammatory neurodegenerative disorders of the human CNS. Front. Neurol. 5:181. doi: 10.3389/fneur.2014.00181

Baranzini, S. E. (2014). Symposium 2-1 the autoimmunome: similarities and differences among genetic susceptibility to common immune-related diseases. Nihon Rinsho Meneki. Gakkai Kaishi. 37:261. doi: 10.2177/jsci.37.261

Cui, J. G., Li, Y. Y., Zhao, Y., Bhattacharjee, S., and Lukiw, W. J. (2010). Differential regulation of interleukin-1 receptor-associated kinase1 and IRAK-2 by miRNA-146a \& NF-kB in human astroglial cells \& in Alzheimer disease. J. Biol. Chem. 285, 38951-38960. doi: 10.1074/jbc.M110.178848

Danborg, P. B., Simonsen, A. H., Waldemar, G., and Heegaard, N. H. (2014). The potential of microRNAs as biofluid markers of neurodegenerative diseases-a systematic review. Biomarkers 19, 259-268. doi: 10.3109/1354750X.2014.904001

Gibson, N. W. (2014). Engineered miRNA therapeutics. J. R. Coll. Physicians Edinb. 44, 196-200. doi: 10.4997/JRCPE. 2014.302

Gilden, D. H. (2005). Infectious causes of multiple sclerosis. Lancet Neurol. 4, 195-202. doi: 10.1016/ S1474-4422(05)01017-3

Gilmore, T. D., and Herscovitch, M. (2006). Inhibitors of NF-kB signaling: 785 and counting. Oncogene 25, 6887-6899. doi: 10.1038/sj.onc.1209982

Gotovac, K., Hajnšek, S., Pašiæ, M. B., Pivac, N., and Boroveèki, F. (2014). Personalized medicine 
in neurodegenerative diseases: how far away? Mol. Diagn. Ther. 18, 17-24. doi: 10.1007/s40291-0130058-z

Gourraud, P. A., Henry, R. G., Cree, B. A., Crane, J. C., Lizee, A., Olson, M. P., et al. (2014). Precision medicine in chronic disease management: the multiple sclerosis BioScreen. Ann. Neurol. 76, 633-642. doi: $10.1002 /$ ana. 24282

Guo, H., Ingolia, N. T., Weissman, J. S., and Bartel, D. P. (2010). Mammalian microRNAs predominantly act to decrease target mRNA levels. Nature 466, 835-840. doi: 10.1038/nature09267

Guo, J., Cheng, C., Yan, W., Xu, G., Feng, J., Wang, T., et al. (2014). Systematic review of clinical practice guidelines related to multiple sclerosis. PLoS ONE 9:e106762. doi: 10.1371/journal.pone. 0106762

Haghikia, A., Haghikia, A., Hellwig, K., Baraniskin, A., Holzmann, A., Décard, B. F., et al. (2012). Regulated microRNAs in the CSF of patients with multiple sclerosis: a casecontrol study. Neurology 79, 2166-2170. doi: 10.1212/WNL.0b013e3182759621

Harris, V. K., and Sadiq, S. A. (2014). Biomarkers of therapeutic response in multiple sclerosis: current status. Mol. Diagn. Ther. 18, 605-617. doi: 10.1007/ s40291-014-0117-0

Hill, J. M., Zhao, Y., Clement, C., Neumann, D. M., and Lukiw, W. J. (2009). HSV-1 infection of human brain cells induces miRNA-146a and Alzheimertype inflammatory signaling. Neuroreport 20, 1500-1505. doi: 10.1097/WNR.0b013e3283329c05

Høglund, R. A., and Maghazachi, A. A. (2014). Multiple sclerosis and the role of immune cells. World J. Exp. Med. 4, 27-37. doi: 10.5493/wjem.v4. i3.27

Ingram, G., Loveless, S., Howell, O. W., Hakobyan, S., Dancey, B., Harris, C. L., et al. (2014). Complement activation in multiple sclerosis plaques: an immunohistochemical analysis. Acta Neuropathol Commun. 2:53. doi: 10.1186/20515960-2-53

Junker, A. (2011). Pathophysiology of translational regulation by microRNAs in multiple sclerosis. FEBS Lett. 585, 3738-3746. doi: 10.1016/j.febslet. 2011.03.052

Junker, A., Krumbholz, M., Eisele, S., Mohan, H., Augstein, F., Bittner, R., et al. (2009). MicroRNA profiling of multiple sclerosis lesions identifies modulators of the regulatory protein CD47. Brain 132, 3342-3352. doi: 10.1093/brain/awp300

Kamphuis, W. W., Troletti, C. D., Reijerkerk, A., Romero, I. A., and de Vries, H. E. (2015). The blood-brain barrier in multiple sclerosis: microRNAs as key regulators. CNS Neurol. Disord. Drug Targets. [Epub ahead of print].

Kroesen, B. J., Teteloshvili, N., Smigielska-Czepiel, K., Brouwer, E., Boots, A. M., van den Berg, A., et al. (2015). Immuno-miRs: critical regulators of T-cell development, function and ageing. Immunology 144, 1-10. doi: 10.1111/imm.12367

Küçükali, C. I., Kürtüncü, M., Coban, A., Cebi, M., and Tüzün, E. (2014). Epigenetics of multiple sclerosis: an updated review. Neuromolecular Med. doi: 10.1007/s12017-014-8298-6. [Epub ahead of print].

Kutty, R. K., Nagineni, C. N., Samuel, W., Vijayasarathy, C., Jaworski, C., Duncan, T., et al. (2013). Differential regulation of miRNA-146a and miRNA-146b-5p in human retinal pigment epithelial cells by IL- $1 \beta$, tumor necrosis factor- $\alpha$, and interferon- $\gamma$. Mol. Vis. 19, 737-750.

Latta, C. H., Brothers, H. M., and Wilcock, D. M (2014). Neuroinflammation in Alzheimer's disease; A source of heterogeneity and target for personalized therapy. Neuroscience. doi: 10.1016/j. neuroscience.2014.09.061. [Epub ahead of print].

Leng, R. X., Pan, H. F., Qin, W. Z., Chen, G. M. and Ye, D. Q. (2011). Role of microRNA-155 in autoimmunity. Cytokine Growth Factor Rev. 22, 141-147. doi: 10.1016/j.cytogfr.2011.05.002

Li, Y. Y., Alexandrov, P. N., Pogue, A. I., Zhao, Y., Bhattacharjee, S., and Lukiw, W. J. (2012). miRNA-155 upregulation and complement factor $\mathrm{H}$ deficits in Down's syndrome. Neuroreport 23, 168-173. doi: 10.1097/WNR.0b013e32834f4eb4

Li, Y. Y., Cui, J. G., Dua, P., Pogue, A. I. Bhattacharjee, S., and Lukiw, W. J. (2011). Differential expression of miRNA-146a-regulated inflammatory genes in human primary neural, astroglial and microglial cells. Neurosci. Lett. 499, 109-113. doi: 10.1016/j.neulet.2011. 05.044

Lopez-Ramirez, M. A., Wu, D., Pryce, G., Simpson, J. E., Reijerkerk, A., King-Robson, J., et al. (2014), MicroRNA- 155 negatively affects blood-brain barrier function during neuroinflammation. FASEB $J$. 28, 2551-2565. doi: 10.1096/fj.13-248880

Lu, F., Weidmer, A., Liu, C. G., Volinia, S., Croce, C. M., and Lieberman, P. M. (2008). Epstein-Barr virus-induced miR-155 attenuates NF-KB signaling and stabilizes latent virus persistence. J. Virol. 82, 10436-10443. doi: 10.1128/JVI.00752-08

Lu, H. E., Yang, Y. C., Chen, S. M., Su, H. L., Huang, P. C., Tsai, M. S., et al. (2013). Modeling neurogenesis impairment in Down syndrome with induced pluripotent stem cells from Trisomy 21 amniotic fluid cells. Exp. Cell Res. 319, 498-505. doi: 10.1016/j.yexcr.2012.09.017

Lukiw, W. J. (2012a). NF-êB-regulated micro RNAs (miRNAs) in primary human brain cells. Exp. Neurol. 235, 484-490. doi: 10.1016/j.expneurol.2011.11.022

Lukiw, W. J. (2012b). NF-кB-regulated, proinflammatory miRNAs in Alzheimer's disease. Alzheimers. Res. Ther. 4, 47. doi: 10.1186/alzrt150

Lukiw, W. J. (2013). Antagonism of NF-kB-upregulated micro RNAs (miRNAs) in sporadic Alzheimer's disease (AD)-anti-NF- $\kappa B$ vs. antimiRNA strategies. Front. Genet. 4:77. doi: 10.3389/fgene.2013.00077

Lukiw, W. J., and Bazan, N. G. (1998). Strong nuclear factor-кB-DNA binding parallels cyclooxygenase2 gene transcription in aging and in sporadic Alzheimer's disease superior temporal lobe neocortex. J. Neurosci. Res. 53, 583-592.

Lukiw, W. J., Cui, J. G., Yuan, L. Y., Bhattacharjee, P. S., Corkern, M., Clement, C., et al. (2010). Acyclovir or A $\beta 42$ peptides attenuate HSV1-induced miRNA-146a levels in human primary brain cells. Neuroreport 21, 922-927. doi: 10.1097/WNR.0b013e32833da51a

Lukiw, W. J., Surjyadipta, B., Dua, P., and Alexandrov, P. N. (2012). Common micro RNAs (miRNAs) target complement factor $\mathrm{H}(\mathrm{CFH})$ regulation in Alzheimer's disease (AD) and in age-related macular degeneration (AMD). Int. J. Biochem. Mol. Biol. $3,105-116$.
Lukiw, W. J., Zhao, Y., and Cui, J. G. (2008). An NF-kB-sensitive micro RNA-146a-mediated inflammatory circuit in Alzheimer disease and in stressed human brain cells. J. Biol. Chem. 283, 31315-31322. doi: 10.1074/jbc. M805371200

Ma, X., Zhou, J., Zhong, Y., Jiang, L., Mu, P., Li, Y., et al. (2014). Expression, regulation and function of microRNAs in multiple sclerosis. Int. J. Med. Sci. 11, 810-818. doi: 10.7150/ijms.8647

Meinl, E., and Meister, G. (2012). MicroRNAs in the CSF: macro-advance in MS? Neurology 79, 2162-2163. doi: 10.1212/WNL.0b013e3182 $7597 \mathrm{~d} 1$

Melcon, M. O., Correale, J., and Melcon, C. M. (2014). Is it time for a new global classification of multiple sclerosis? J. Neurol. Sci. 344, 171-181. doi: 10.1016/j.jns.2014.06.051

Murugaiyan, G., Beynon, V., Mittal, A., Joller, N., and Weiner, H. L. (2011). Silencing microRNA155 ameliorates experimental autoimmune encephalomyelitis. J. Immunol. 187, 2213-2221. doi: 10.4049/jimmunol.1003952

O'Connell, R. M., Kahn, D., Gibson, W. S., Round, J. L., Scholz, R. L., Chaudhuri, A. A., et al. (2010). MicroRNA-155 promotes autoimmune inflammation by enhancing inflammatory $\mathrm{T}$ cell development. Immunity 33, 607-619. doi: 10.1016/j.immuni.2010.09.009

Pogue, A. I., Li, Y. Y., Cui, J. G., Zhao, Y., Kruck, T. P., Percy, M. E., et al. (2009). Characterization of an NF-kB-regulated, miRNA-146a-mediated downregulation of $\mathrm{CFH}$ in metal-sulfate-stressed human brain cells. J. Inorg. Biochem. 103, 1591-1595. doi: 10.1016/j.jinorgbio.2009.05.012

Saba, R., Gushue, S., Huzarewich, R. L., Manguiat, K., Medina, S., Robertson, C., et al. (2012). miRNA-146a is over-expressed during prion disease and modulates the innate immune response and the microglial activation state. PLoS ONE 7:e30832. doi: 10.1371/journal.pone. 0030832

Seddiki, N., Brezar, V., Ruffin, N., Lévy, Y., and Swaminathan, S. (2014). Role of miR-155 in the regulation of lymphocyte immune function and disease. Immunology 142, 32-38. doi: 10.1111/ imm. 12227

Sethi, P., and Lukiw, W. J. (2009). Micro-RNA abundance and stability in human brain: specific alterations in Alzheimer's disease temporal lobe neocortex. Neurosci. Lett. 459, 100-104. doi: 10.1016/j.neulet.2009.04.052

Sturm, D., Gurevitz, S. L., and Turner, A. (2014) Multiple sclerosis: a review of the disease and treatment options. Consult. Pharm. 29, 469-479. doi: 10.4140/TCP.n.2014.469

Stürner, K. H., Verse, N., Yousef, S., Martin, R., and Sospedra, M. (2014). Boswellic acids reduce Th17 differentiation via blockade of IL-1 $\beta$-mediated IRAK1 signaling. Eur. J. Immunol. 44, 1200-1212. doi: $10.1002 /$ eji.201343629

Taganov, K. D., Boldin, M. P., Chang, K. J., and Baltimore, D. (2006). NF-kB-dependent induction of microRNA miR-146, an inhibitor targeted to signaling proteins of innate immune responses. Proc. Natl. Acad. Sci. U.S.A. 103, 12481-12486. doi: 10.1073/pnas.0605298103

Thamilarasan, M., Koczan, D., Hecker, M., Paap, B., and Zettl, U. K. (2012). MicroRNAs in 
multiple sclerosis and experimental autoimmune encephalomyelitis. Autoimmun. Rev. 11, 174-179. doi: 10.1016/j.autrev.2011.05.009

Zhang, J., Cheng, Y., Cui, W., Li, M., Li, B., and Guo, L. (2014). MiRNA-155 modulates Th1 and Th17 cell differentiation and is associated with MS and EAE. J. Neuroimmunol. 266, 56-63. doi: 10.1016/j.jneuroim.2013.09.019

Zhao, Y., Bhattacharjee, S., Jones, B. M., Hill, J., Dua, P., and Lukiw, W. J. (2014). Regulation of neurotropic signaling by the inducible, NF-kBsensitive miRNA-125b in Alzheimer's disease (AD) and in primary human neuronal-glial
(HNG) cells. Mol. Neurobiol. 50, 97-106. doi: 10.1007/s12035-013-8595-3

Conflict of Interest Statement: The authors declare that the research was conducted in the absence of any commercial or financial relationships that could be construed as a potential conflict of interest.

Received: 27 October 2014; accepted: 12 February 2015; published online: 02 March 2015.

Citation: Devier DJ, Lovera JF and Lukiw WJ (2015) Increase in NF- $\kappa$ B-sensitive miRNA-146a and miRNA155 in multiple sclerosis (MS) and pro-inflammatory neurodegeneration. Front. Mol. Neurosci. 8:5. doi: 10.3389/fnmol.2015.00005

This article was submitted to the journal Frontiers in Molecular Neuroscience.

Copyright (c) 2015 Devier, Lovera and Lukiw. This is an open-access article distributed under the terms of the Creative Commons Attribution License (CC BY). The use, distribution or reproduction in other forums is permitted, provided the original author(s) or licensor are credited and that the original publication in this journal is cited, in accordance with accepted academic practice. No use, distribution or reproduction is permitted which does not comply with these terms. 